\title{
Sharp for SARP: Nonparametric bounds on counterfactual demands.
}

\author{
Richard Blundell; Martin Browning; Laurens Cherchye \\ Ian Crawford, Bram De Rock ${ }^{\uparrow}$ and Frederic Vermeulen"
}

January 2014

\begin{abstract}
Sharp nonparametric bounds are derived for counterfactual demands and Hicksian compensating and equivalent variations. These "i-bounds" refine and extend earlier results of Blundell, Browning and Crawford (2008). We show that their bounds are sharp under the Weak Axiom of Revealed Preference (WARP) since they do not require transitivity. The new bounds are sharp under the Strong Axiom of Revealed Preference (SARP). By requiring transitivity they can be used to bound welfare measures. The new bounds on welfare measures are shown to be operationalized through algorithms that are easy to implement.
\end{abstract}

\section{Introduction}

Demand analysis is a powerful tool for the measurement of the behaviour and distributional effects of counterfactual price and income changes. A policy maker may, for example, be interested in the impact on the consumer's well-being of an introduction of a tax on the fat content of food or of a change in the indirect taxes on gasoline. The common characteristic of such taxes is that they change the relative prices faced by the consumer. How the consumer reacts to this, by choosing an alternative consumption bundle, is subject of the analysis of demand behaviour. Typically the researcher estimates the unknown parameters of a parametric demand system and uses these estimates to calculate pre- and post-reform demands and associated indirect utilities (see, for example, Banks, Blundell and Lewbel, 1997). Comparing these indirect utilities then allows the econometrician to evaluate the impact of the policy reform on the consumer's

*Department of Economics, University College of London, Gower Street, London WC1 E6BT, United Kingdom. E-mail: r.blundell@ucl.ac.uk.

${ }^{\dagger}$ Department of Economics, University of Oxford, Manor Road Building, Manor Road, Oxford OX1 3UQ, United Kingdom. E-mail: martin.browning@economics.ox.ac.uk.

$\ddagger$ Center for Economic Studies, University of Leuven. E. Sabbelaan 53, B-8500 Kortrijk, Belgium. E-mail: laurens.cherchye@kuleuven-kortrijk.be. Laurens Cherchye gratefully acknowledges financial support from the Research Fund K.U.Leuven through the grant STRT1/08/004.

${ }^{\S}$ Department of Economics, University of Oxford, Manor Road Building, Manor Road, Oxford OX1 3UQ, United Kingdom. E-mail: ian.crawford@economics.ox.ac.uk.

IECARES, Université Libre de Bruxelles. Avenue F.D. Roosevelt 50, 1050 Brussels, Belgium. Email: bderock@ulb.ac.be. Bram De Rock gratefully acknowledges the European Research Council (ERC) for his Starting Grant.

" Department of Economics, University of Leuven, Naamsestraat 69, B-3000 Leuven, Belgium. Email: frederic.vermeulen@kuleuven.be. Frederic Vermeulen gratefully acknowledges financial support from the Research Fund KU Leuven through the grant STRT/12/001. 
well-being. One particularly useful cardinalization of the indirect utility function is Samuelson's (1974) money metric indirect utility function, which allows one to express the change in well-being in monetary units. Depending on the base price that is used in the analysis, this approach leads to the well-known compensating variation (base price equals the post-reform prices) and equivalent variation (base price equals pre-reform prices) that were proposed by Hicks (1939).

A major disadvantage of standard demand and welfare analyses is that they rely on the functional specification of the indirect utility function that is used. An alternative way to analyze policy reforms is based on the revealed preference (RP) approach, whose foundations were laid down by Samuelson (1938, 1948), Houthakker (1950), Afriat (1967), Diewert (1973) and Varian (1982). The RP approach translates conditions for rational consumption behaviour into testable implications. These do not depend on any assumptions about the specification of the consumer's demand system or the particular representation of her rational preferences. The major disadvantage of the RP approach, however, is that the predictions of demand responses derived from its restrictions are set-valued, i.e. it is only possible to recover bounds on predicted demands.

As a response Blundell, Browning and Crawford (2003, 2008) proposed blending these two approaches by combining Engel curve estimation with RP conditions. This has shown to be a productive technique. Firstly, it makes the RP conditions applicable to the types of datasets which are widely available to researchers (such as the Family Expenditure Survey from the UK or the Consumption Expenditure Survey from the US). Secondly, the approach is easy to implement and therefore contributes to the practical usefulness of RP conditions. Finally, and principally, it allows for empirical RP analysis with substantial discriminatory and forecasting power. ${ }^{1}$

However, whilst Blundell, Browning and Crawford (2008) showed how to improve bounds on the demand responses to price changes, they did so without fully exploiting all of the empirical implications of rational preferences. We show that their bounds are (only) sharp under the Weak Axiom of Revealed Preference (WARP). In contrast to what is claimed, the bounds do not impose all the restrictions of transitivity. Further improvements are in general possible if preferences can also be assumed to satisfy transitivity. $^{2}$ That is, if preferences satisfy the Strong Axiom of Revealed Preference (SARP).

For welfare calculations transitivity is, in general, required. This is because nontransitivity can lead to cycles and path-dependence if one attempts to integrate back to utility constant welfare measures. In this paper, we refine and extend the results of Blundell, Browning and Crawford $(2003,2008)$ by fully exploiting transitivity. This derives sharp bounds on counterfactual demand responses and welfare calculations under SARP and as such these bounds are "sharp for SARP". 3 For reasons which will become clear we refer to these bounds as "iterated bounds", or $i$-bounds.

Next, we show how our method can be used to provide sharp nonparametric bounds on compensating and equivalent variations. For doing so, we provide easy-to-implement algorithms that allow computing tightest (iterated) bounds on Marshallian demands. This in turn obtains sharp bounds for compensating and equivalent variations.

As a final contribution, we illustrate our results on data also used by Blundell, Browning and Crawford (2008). We show that even in a simple set-up our i-bounds significantly improve upon the bounds defined by Blundell, Browning and Crawford (2008). We also demonstrate the easy application of our algorithms to compute sharp

\footnotetext{
${ }^{1}$ See Blundell (2005), Blundell, Browning and Crawford (2007) and Blundell, Kristensen and Matzkin (2013) for recent contributions that build further on the basic insights of Blundell, Browning and Crawford $(2003,2008)$. Blundell, Kristensen and Matzkin (2013) is notable since it applies this approach to quantile demands and allows for unobserved heterogeneity in individual demands.

${ }^{2}$ Note that in situations in which there are only two goods transitivity adds no further restrictions to WARP (see Rose, 1958).

${ }^{3}$ In terms of RP restrictions, the recovery of demand responses under Walras' Law, homogeneity of degree zero and negative semi-definiteness of the Slutsky matrix is equivalent to imposition of WARP, whereas the requirement that these demands are consistent with full rationality amounts to SARP (see Kihlstrom, Mas-Colell and Sonnenschein, 1976).
} 
bounds on compensating and equivalent variations. Evidently, bringing this method to observational data in a more thorough way necessarily requires dealing with empirical issues such as measurement error and (un)observed heterogeneity. For compactness, we abstract from a detailed discussion in the current paper. Blundell, Browning and Crawford (2003, 2008) and Blundell, Kristensen and Matzkin (2013) propose methodological extensions for dealing with these issues that are directly applicable to the method we introduce below.

The rest of the paper unfolds as follows. In Section 2, we introduce our iterated bounds on the Marshallian demands for any number of goods, and we provide an easily implementable method for computing these bounds. In Section 3, we introduce the corresponding method for identifying the tightest bounds on compensating and equivalent variations. Section 4 contains our empirical illustration and Section 5 concludes.

\section{Iterated bounds on Marshallian demands}

To set the stage, we first briefly recall the concept of e-bounds introduced by Blundell, Browning and Crawford (2008, henceforth BBC (2008)). Subsequently, we take the sequential maximum power path idea for constructing bounds on welfare measures developed in Blundell, Browning and Crawford (2003, BBC (2003)) and use this to introduce the notion of iterated bounds on Marshallian demands. We present an example to demonstrate that these bounds can be used to improve upon the e-bounds if there are more than two goods. Given this result, we next show that our iterated bounds procedure leads to tightest bounds on Marshallian demands. We end this section by presenting an algorithm to compute the iterated bounds. As we will indicate, this algorithm essentially iterates a procedure originally proposed by BBC (2003), which explains the name "iterated bounds".

\section{1 e-bounds}

We assume $J$ goods and consider a consumer with a (nonnegative) Marshallian demand function $\mathbf{q}(\mathbf{p}, x)$ for prices $\mathbf{p} \in \mathbb{R}_{++}^{J}$ and income $x \in \mathbb{R}_{++}$. Following BBC (2008), we assume uniqueness of demands.

Assumption 1 (uniqueness of demands) The demand function $\mathbf{q}(\mathbf{p}, x): \mathbb{R}_{++}^{J+1} \rightarrow$ $\mathbb{R}_{+}^{J}$ satisfies adding up, i.e. $\mathbf{p}^{\prime} \mathbf{q}(\mathbf{p}, x)=x$ for all prices $\mathbf{p}$ and incomes $x$.

Consider a set of $T$ price vectors $\left\{\mathbf{p}_{t}\right\}_{t=1, \ldots, T}$; we say there are $T$ observations. For a given price vector $\mathbf{p}_{t}$ we denote the $J$-valued demand associated with income $x$ as $\mathbf{q}_{t}(x)$, and we refer to the function $\mathbf{q}_{t}$ as the expansion path that corresponds to the prices $\mathbf{p}_{t}$. Again, we follow BBC (2008) by assuming weak normality of $\mathbf{q}_{t}$.

Assumption 2 (weak normality) If $x>\bar{x}$, then $\mathbf{q}_{t}(x) \geq \mathbf{q}_{t}(\bar{x})$ for all $\mathbf{p}_{t}$.

BBC (2008) address the following question: "Given a new budget $\left\{\mathbf{p}_{N}, x_{N}\right\}$ and a set of observed prices and expansion paths $\left\{\mathbf{p}_{t}, \mathbf{q}_{t}(x)\right\}_{t=1, \ldots, T}$, what values of $\mathbf{q}_{N}$, which exhaust the budget (i.e. $\mathbf{p}_{N}^{\prime} \mathbf{q}_{N}=x_{N}$ ), are consistent with these observed demands and utility maximization?" Let us denote the bundles that exhaust the new budget $\left\{\mathbf{p}_{N}, x_{N}\right\}$ by $B\left(\mathbf{p}_{N}, x_{N}\right)=\left\{\mathbf{q}_{N} \in \mathbb{R}_{+}^{J} \mid \mathbf{p}_{N}^{\prime} \mathbf{q}_{N}=x_{N}\right\}$.

To state BBC (2008)'s answer to their question, we first need to introduce some revealed preferences (RP) concepts. We start by defining direct revealed preference relations $R^{0}$.

Definition 1 (direct revealed preference) If at prices $\mathbf{p}_{t}$ and income $x_{t}$ the consumer chooses $\mathbf{q}_{t}\left(x_{t}\right)$ and $\mathbf{p}_{t}^{\prime} \mathbf{q}_{t}\left(x_{t}\right) \geq \mathbf{p}_{t}^{\prime} \mathbf{q}_{s}\left(x_{s}\right)$, then $\mathbf{q}_{t}\left(x_{t}\right) R^{0} \mathbf{q}_{s}\left(x_{s}\right)$.

Transitivity of preferences then leads to the next concept of indirect revealed preference relations $R$. 
Definition 2 (indirect revealed preference) If we have a sequence $\mathbf{q}_{t}\left(x_{t}\right) R^{0} \mathbf{q}_{u}\left(x_{u}\right)$ $R^{0} \mathbf{q}_{v}\left(x_{v}\right) \cdots R^{0} \mathbf{q}_{w}\left(x_{w}\right) R^{0} \mathbf{q}_{s}\left(x_{s}\right)$, then $\mathbf{q}_{t}\left(x_{t}\right) R \mathbf{q}_{s}\left(x_{s}\right)$.

In our following exposition, we will consider two consistency conditions for utility maximizing consumer behaviour: the Weak Axiom of Revealed Preference (WARP) and the Strong Axiom of Revealed Preference (SARP). It is well-known that SARP is a necessary and sufficient condition for utility maximization, while WARP is only a necessary condition (see Varian, 1982 and 2006, for a detailed discussion).

\section{Definition 3 (WARP and SARP)}

(i) The demands $\mathbf{q}_{t}\left(x_{t}\right), t=1, \ldots, T$, satisfy WARP if $\mathbf{q}_{t}\left(x_{t}\right) R^{0} \mathbf{q}_{s}\left(x_{s}\right)$ and $\mathbf{q}_{t}\left(x_{t}\right) \neq$ $\mathbf{q}_{s}\left(x_{s}\right)$ then not $\mathbf{q}_{s}\left(x_{s}\right) R^{0} \mathbf{q}_{t}\left(x_{t}\right)$ for any $s$ and $t$.

(ii) The demands $\mathbf{q}_{t}\left(x_{t}\right), t=1, \ldots, T$, satisfy SARP if $\mathbf{q}_{t}\left(x_{t}\right) R \mathbf{q}_{s}\left(x_{s}\right)$ and $\mathbf{q}_{t}\left(x_{t}\right) \neq$ $\mathbf{q}_{s}\left(x_{s}\right)$ then not $\mathbf{q}_{s}\left(x_{s}\right) R^{0} \mathbf{q}_{t}\left(x_{t}\right)$ for any $s$ and $t$.

Because WARP only uses direct revealed preference relations $R^{0}$, while SARP focuses on indirect revealed preference relations $R$ (so exploiting transitivity of preferences), we obtain that SARP is a stronger condition than WARP. As in BBC (2008), we will assume that the expansion paths $\mathbf{q}_{t}(x)$ generate demands that are consistent with utility maximization. In RP terms, this implies the following assumption.

Assumption 3 (SARP) For all $x \in \mathbb{R}_{++}$, the demands $\mathbf{q}_{t}(x), t=1, \ldots, T$ satisfy SARP.

To formalize their notion of e-bounds, BBC (2008) use the concept of intersection demands. To facilitate our following comparison of BBC (2008)'s e-bounds with our iterated bounds, we here introduce these intersection demands in a slightly different way, i.e. in terms of intersection incomes.

Definition 4 (intersection income) The intersection income $\tilde{x}_{t}$, for $t \in\{1, \ldots, T\}$, is the maximal income for which

$$
\forall \mathbf{q}_{N} \in B\left(\mathbf{p}_{N}, x_{N}\right): \mathbf{q}_{N} R^{0} \mathbf{q}_{t}\left(\tilde{x}_{t}\right) .
$$

The assumptions of uniqueness and normality ensure that each intersection income $\tilde{x}_{t}$ is uniquely defined. More precisely, it is the income level such that $\mathbf{p}_{N}^{\prime} \mathbf{q}_{t}\left(\tilde{x}_{t}\right)=x_{N}$. BBC (2008) refer to the corresponding value of the expansion path, $\mathbf{q}_{t}\left(\tilde{x}_{t}\right)$, as the intersection demand for observation $t$.

Given all this, BBC (2008) define the support set

$$
S^{B B C}\left(\mathbf{p}_{N}, x_{N}\right)=\left\{\mathbf{q}_{N}: \begin{array}{c}
\mathbf{q}_{N} \in B\left(\mathbf{p}_{N}, x_{N}\right) \\
\left\{\mathbf{p}_{N}, \mathbf{p}_{t} ; \mathbf{q}_{N}, \mathbf{q}_{t}\left(\tilde{x}_{t}\right)\right\}_{t=1, \ldots, T} \text { satisfy SARP }
\end{array}\right\} ;
$$

and they label the bounds on demand responses that are based on $S^{B B C}\left(\mathbf{p}_{N}, x_{N}\right)$ as e-bounds.

To end this section, we present a specific characterization of the support set $S^{B B C}\left(\mathbf{p}_{N}\right.$, $\left.x_{N}\right)$. As we will explain, this characterization will directly motivate our following research question, i.e. define "iterated bounds" that improve upon the e-bounds. Essentially, the next proposition distinguishes between two cases for $\mathbf{q}_{N} \in S^{B B C}\left(\mathbf{p}_{N}, x_{N}\right)$ : either $\mathbf{q}_{N}$ is different from the intersection demand $\mathbf{q}_{t}\left(\tilde{x}_{t}\right)$ for any observation $t$, or we have $\mathbf{q}_{N}=\mathbf{q}_{s}\left(\tilde{x}_{s}\right)$ for some observation $s .{ }^{4}$ Appendix A contains the proofs of all our results.

\footnotetext{
${ }^{4}$ Note that we make the (implicit) assumption that every observation $t$ corresponds to a different intersection demand $\mathbf{q}_{t}\left(\tilde{x}_{t}\right)$. Dropping this assumption is actually straightforward, but it would substantially complicate the statement of Proposition 1 without really adding new insights. A similar qualification applies to Theorem 1 .
} 
Proposition 1 (profitable characterization of $S^{B B C}\left(\mathbf{p}_{N}, x_{N}\right)$ ) For any $\mathbf{q}_{N}$ in the budget set $B\left(\mathbf{p}_{N}, x_{N}\right)$, we have that $\mathbf{q}_{N}$ is in the support set $S^{B B C}\left(\mathbf{p}_{N}, x_{N}\right)$ (i.e. meets the e-bounds) if and only if

(i) $\forall t \in\{1, \ldots, T\}: \mathbf{p}_{t}^{\prime} \mathbf{q}_{t}\left(\tilde{x}_{t}\right)<\mathbf{p}_{t}^{\prime} \mathbf{q}_{N}$, or

(ii) $\exists s \in\{1, \ldots, T\}: \mathbf{q}_{N}=\mathbf{q}_{s}\left(\tilde{x}_{s}\right)$, and then $\mathbf{p}_{t}^{\prime} \mathbf{q}_{t}\left(\tilde{x}_{t}\right)<\mathbf{p}_{t}^{\prime} \mathbf{q}_{N}$ for all $t \in\{1, \ldots T\} \backslash\{s\}$.

Inspection of Proposition 1 reveals that the definition of e-bounds nowhere exploits transitivity of preferences, which is captured by the indirect revealed preference relations $R$. Specifically, any $\mathbf{q}_{N} \in S^{B B C}\left(\mathbf{p}_{N}, x_{N}\right)$ can be characterized in terms of direct revealed preference relations $R^{0}$, i.e. it satisfies

$$
\mathbf{p}_{N}^{\prime} \mathbf{q}_{t}\left(\tilde{x}_{t}\right)=x_{N}=\mathbf{p}_{N}^{\prime} \mathbf{q}_{N} \text { (i.e. } \mathbf{q}_{N} R^{0} \mathbf{q}_{t}\left(\tilde{x}_{t}\right) \text { ), }
$$

which follows from the definition of the intersection demands, and

$$
\left.\mathbf{p}_{t}^{\prime} \mathbf{q}_{t}\left(\tilde{x}_{t}\right)<\mathbf{p}_{t}^{\prime} \mathbf{q}_{N} \text { (i.e. } \operatorname{not} \mathbf{q}_{t}\left(\tilde{x}_{t}\right) R^{0} \mathbf{q}_{N}\right)
$$

which follows from Proposition 1. Putting it differently, e-bounds only use the empirical restrictions that are implied by WARP consistency. However, as indicated above, utility maximizing behaviour requires SARP consistency, which generally involves further restrictions than WARP consistency. ${ }^{5}$ Therefore, in what follows we will define iterated bounds that do fully exploit the restrictions implied by transitivity of preferences. Essentially, this will require generalizations of the concepts intersection income and intersection demand that are based on the relations $R$ (instead of $R^{0}$ ).

\section{2 i-bounds}

We define iterated bounds, or i-bounds, as bounds on demand responses based on a support set $S\left(\mathbf{p}_{N}, x_{N}\right)$ that accounts for all possible incomes $x_{t}$ (rather than only $\tilde{x}_{t}$ ), i.e.

$$
S\left(\mathbf{p}_{N}, x_{N}\right)=\left\{\mathbf{q}_{N}: \begin{array}{c}
\mathbf{q}_{N} \in B\left(\mathbf{p}_{N}, x_{N}\right) \\
\left\{\mathbf{p}_{N}, \mathbf{p}_{t} ; \mathbf{q}_{N}, \mathbf{q}_{t}\left(x_{t}\right)\right\}_{t=1, \ldots, T ; x_{t} \in \mathbb{R}_{++}} \text {satisfy SARP }
\end{array}\right\} .
$$

Because this set $S\left(\mathbf{p}_{N}, x_{N}\right)$ considers all demands on the expansion paths $\mathbf{q}_{t}$, it is the tightest (i.e. smallest) SARP-based support set by construction. In turn, this implies that i-bounds are tightest bounds on demand responses. However, as it is formulated here, the set $S\left(\mathbf{p}_{N}, x_{N}\right)$ is not directly useful from a practical point of view: for each $t$, it requires considering infinitely many points on every expansion path. To derive an operational characterization of $S\left(\mathbf{p}_{N}, x_{N}\right)$, we will make use of the following notion of most informative income.

Definition 5 (most informative income) The most informative income $\widehat{x}_{t}$, for $t \in$ $\{1, \ldots, T\}$, is the maximal income for which

$$
\forall \mathbf{q}_{N} \in B\left(\mathbf{p}_{N}, x_{N}\right): \mathbf{q}_{N} R \mathbf{q}_{t}\left(\widehat{x}_{t}\right),
$$

i.e. there exist $x_{u}, x_{v}, \ldots, x_{w}$ such that $\mathbf{q}_{N} R^{0} \mathbf{q}_{u}\left(x_{u}\right) R^{0} \mathbf{q}_{v}\left(x_{v}\right) \ldots R^{0} \mathbf{q}_{w}\left(x_{w}\right) R^{0}$ $\mathbf{q}_{t}\left(\widehat{x}_{t}\right)$.

This concept of most informative income extends the earlier notion of intersection income by using indirect revealed preference relations $R$ instead of (only) direct revealed preference relations $R^{0}$. Because the relations $R$ include the relations $R^{0}$ by construction, we obtain $\widehat{x}_{t} \geq \tilde{x}_{t}$. As before, the assumptions of uniqueness and weak normality

\footnotetext{
${ }^{5}$ In this respect, one may also state that e-bounds are best WARP-based bounds but not best SARP-based bounds.
} 
imply that most informative incomes $\widehat{x}_{t}$ are uniquely defined. However, in contrast to intersection incomes, there is no closed formula for computing most informative incomes. Fortunately, as we will discuss in Section 2.3, we can define an easy-to-implement (finite and efficient) algorithm to compute $\widehat{x}_{t}$ by iterating the procedure for computing the intersection incomes. Analogous to before, we will refer to the associated value of the expansion path, $\mathbf{q}_{t}\left(\widehat{x}_{t}\right)$, as the most informative demand for observation $t$.

The next result provides a characterization of the set $S\left(\mathbf{p}_{N}, x_{N}\right)$ that parallels the one of $S^{B B C}\left(\mathbf{p}_{N}, x_{N}\right)$ in Proposition 1. It also provides a specific definition of $S\left(\mathbf{p}_{N}, x_{N}\right)$ in terms of the most informative incomes $\widehat{x}_{t}$. In practical applications, this allows for constructing the set $S\left(\mathbf{p}_{N}, x_{N}\right)$ once these most informative incomes have been identified.

Theorem 1 For any $\mathbf{q}_{N}$ in the budget set $B\left(\mathbf{p}_{N}, x_{N}\right)$, we have that $\mathbf{q}_{N}$ is in the support set $S\left(\mathbf{p}_{N}, x_{N}\right)$ (i.e. meets the $i$-bounds) if and only if

(i) $\forall t \in\{1, \ldots, T\}: \mathbf{p}_{t}^{\prime} \mathbf{q}_{t}\left(\widehat{x}_{t}\right)<\mathbf{p}_{t}^{\prime} \mathbf{q}_{N}$, or

(ii) $\exists s \in\{1, \ldots, T\}: \mathbf{q}_{N}=\mathbf{q}_{s}\left(\widehat{x}_{s}\right)$ and then $\mathbf{p}_{t}^{\prime} \mathbf{q}_{t}\left(\widehat{x}_{t}\right)<\mathbf{p}_{t}^{\prime} \mathbf{q}_{N}$ for all $t \in\{1, \ldots T\} \backslash\{s\}$.

We conclude this section by Example 1, which demonstrates that BBC (2008)'s support set $S^{B B C}\left(\mathbf{p}_{N}, x_{N}\right)$ (yielding e-bounds on demand responses) need not coincide with the smallest SARP-based support set $S\left(\mathbf{p}_{N}, x_{N}\right)$ (yielding iterated or tightest bounds on demand responses). The example also illustrates the central intuition behind this result. Specifically, it presents expansion paths where, for some $t(t=1$ in Example 1 ), the most informative income $\widehat{x}_{t}$ is strictly above the intersection income $\tilde{x}_{t}$, which implies that there exists $\mathbf{q}_{N}$ with $\mathbf{q}_{N} R \mathbf{q}_{t}\left(\widehat{x}_{t}\right)$ but not $\mathbf{q}_{N} R^{0} \mathbf{q}_{t}\left(\widehat{x}_{t}\right)$. Because the set $S\left(\mathbf{p}_{N}, x_{N}\right)$ must satisfy SARP, this yields the restriction $\mathbf{p}_{t}^{\prime} \mathbf{q}_{t}\left(\widehat{x}_{t}\right)<\mathbf{p}_{t}^{\prime} \mathbf{q}_{N}$, which is stronger than $\mathbf{p}_{t}^{\prime} \mathbf{q}_{t}\left(\tilde{x}_{t}\right)<\mathbf{p}_{t}^{\prime} \mathbf{q}_{N}$ (because $\widehat{x}_{t}>\tilde{x}_{t}$ ). In turn, this effectively excludes from the set $S\left(\mathbf{p}_{N}, x_{N}\right)$ some $\mathbf{q}_{N}$ that belongs to the set $S^{B B C}\left(\mathbf{p}_{N}, x_{N}\right)$. This demonstrates that, in general, we can have $S\left(\mathbf{p}_{N}, x_{N}\right) \subsetneq S^{B B C}\left(\mathbf{p}_{N}, x_{N}\right)$.

As a final note, we emphasize that we need more than two goods for $S\left(\mathbf{p}_{N}, x_{N}\right) \subsetneq$ $S^{B B C}\left(\mathbf{p}_{N}, x_{N}\right)$. Indeed, as indicated above, the support set $S^{B B C}\left(\mathbf{p}_{N}, x_{N}\right)$ exploits the empirical restrictions implied by WARP consistency. And it is well-known that WARP and SARP have the same empirical content if there are only two goods (see Rose, 1958), so that we always get $S\left(\mathbf{p}_{N}, x_{N}\right)=S^{B B C}\left(\mathbf{p}_{N}, x_{N}\right)$ in this case.

Example 1 We consider the support set $S^{B B C}\left(\mathbf{p}_{N}, x_{N}\right)$ for $\mathbf{p}_{N}=(3,2,4)$ and $x_{N}=$ 15. Suppose we observe two expansion paths $\mathbf{q}_{1}$ and $\mathbf{q}_{2}$, which are associated with the prices $\mathbf{p}_{1}=(4,3,2)$ and $\mathbf{p}_{2}=(2,4,3)$.

Suppose we have the intersection incomes $\tilde{x}_{1}=13.5$ and $\tilde{x}_{2}=15.8$, with corresponding intersection demands

$$
\mathbf{q}_{1}\left(\tilde{x}_{1}\right)=(2,0.5,2) \text { and } \mathbf{q}_{2}\left(\tilde{x}_{2}\right)=(2.3,2.05,1) .
$$

Next, we assume the following most informative incomes. Let $\widehat{x}_{1}=15>\tilde{x}_{1}$, with

$$
\mathbf{q}_{1}\left(\widehat{x}_{1}\right)=(2,1,2),
$$

while $\widehat{x}_{2}=\widetilde{x}_{2}$ and, thus, $\mathbf{q}_{2}\left(\tilde{x}_{2}\right)=\mathbf{q}_{2}\left(\widehat{x}_{2}\right)$. We remark that an expansion path $\mathbf{q}_{1}\left(x_{1}\right)$ containing both $\mathbf{q}_{1}\left(\widehat{x}_{1}\right)$ and $\mathbf{q}_{1}\left(\tilde{x}_{1}\right)$ does not conflict with our earlier assumptions.

We can then show that $S\left(\mathbf{p}_{N}, x_{N}\right) \subsetneq S^{B B C}\left(\mathbf{p}_{N}, x_{N}\right)$. To obtain the result, it suffices to show that there exists $\mathbf{q}_{N}$ with

$$
\mathbf{q}_{N} \in S^{B B C}\left(\mathbf{p}_{N}, x_{N}\right) \text { and } \mathbf{q}_{N} \notin S\left(\mathbf{p}_{N}, x_{N}\right) .
$$

For the current example, this applies to $\mathbf{q}_{N}=(1,2,2)$ (which effectively meets $\mathbf{p}_{N}^{\prime} \mathbf{q}_{N}=$ $\left.x_{N}\right)$. First, we can verify that $\mathbf{q}_{N} \in S^{B B C}\left(\mathbf{p}_{N}, x_{N}\right)$ : the demands $\mathbf{q}_{N}, \mathbf{q}_{1}\left(\tilde{x}_{1}\right), \mathbf{q}_{2}\left(\tilde{x}_{2}\right)$ satisfy SARP (with $\mathbf{q}_{N} R^{0} \mathbf{q}_{2}\left(\tilde{x}_{2}\right) R^{0} \mathbf{q}_{1}\left(\tilde{x}_{1}\right)$ ). On the other hand, we also obtain $\mathbf{q}^{0} \notin S\left(\mathbf{p}_{N}, x_{N}\right)$ : the demands $\mathbf{q}_{N}, \mathbf{q}_{1}\left(\widehat{x}_{1}\right), \mathbf{q}_{2}\left(\widehat{x}_{2}\right)$ do not meet $S A R P$, which a fortiori 
implies $\mathbf{q}_{N} \notin S\left(\mathbf{p}_{N}, x_{N}\right)$; in particular, we get $\mathbf{q}_{N} R^{0} \mathbf{q}_{2}\left(\widehat{x}_{2}\right) R^{0} \mathbf{q}_{1}\left(\widehat{x}_{1}\right) R^{0} \mathbf{q}_{N} .{ }^{6}$

\title{
2.3 An algorithm for computing most informative incomes
}

The following algorithm uses the approach in BBC (2003) to define the most informative incomes $\hat{x}_{1}, \ldots, \hat{x}_{T}$ and, thus, also the corresponding demands $\mathbf{q}_{1}\left(\hat{x}_{1}\right), \ldots, \mathbf{q}_{T}\left(\hat{x}_{T}\right)$.

\begin{abstract}
Algorithm 1 (computing most informative incomes)
Input: $\left\{\mathbf{p}_{N}, x_{N}\right\}$ and $\left\{\mathbf{p}_{1}, \ldots, \mathbf{p}_{T} ; \mathbf{q}_{1}\left(x_{1}\right), \ldots, \mathbf{q}_{T}\left(x_{T}\right)\right\}$.

Output: $\hat{x}_{1}, \ldots, \hat{x}_{T}$.

Step 0: Set $s=0$ and $F_{s}=\left\{x_{1}, \ldots, x_{T} \mid \mathbf{p}_{N}^{\prime} \mathbf{q}_{1}\left(x_{1}\right)=x_{N}, \ldots, \mathbf{p}_{N}^{\prime} \mathbf{q}_{T}\left(x_{T}\right)=x_{N}\right\}$.

Step 1: Set $F_{s+1}=\left\{\max _{x_{t} \in F_{s}}\left(x: x_{t}=\mathbf{p}_{t}^{\prime} \mathbf{q}_{1}(x)\right), \ldots, \max _{x_{t} \in F_{s}}\left(x: x_{t}=\mathbf{p}_{t}^{\prime} \mathbf{q}_{T}(x)\right)\right\}$.

Step 2: If $F_{s+1} \equiv F_{s}$ then set $\left\{\hat{x}_{1}, \ldots, \hat{x}_{T}\right\}=F_{s+1}$ and stop. Else set $s=s+1$ and go to Step 1.
\end{abstract}

Note that Step 0 of this algorithm delivers the intersection incomes $\tilde{x}_{t}$, which BBC (2008) originally considered to define their e-bounds on Marshallian demands. To define our most informative incomes $\widehat{x}_{t}$ (and so i-bounds on Marshallian demands), we iterate this procedure in Steps 1 and 2. This iteration implies that most informative incomes may effectively exceed intersection incomes (i.e. $\widehat{x}_{t}>\tilde{x}_{t}$ ). As explained in our discussion of Example 1, such an instance effectively obtains $S\left(\mathbf{p}_{N}, x_{N}\right) \subsetneq S^{B B C}\left(\mathbf{p}_{N}, x_{N}\right)$.

The following lemma states two important properties of Algorithm 1.

\section{Lemma 1}

(i) Algorithm 1 converges in a finite number of steps.

(ii) For any $x_{t}$ we have $\mathbf{q}_{t}\left(\hat{x}_{t}\right) \geq \mathbf{q}_{t}\left(x_{t}\right) \Leftrightarrow \mathbf{q}_{N} R \mathbf{q}_{t}\left(x_{t}\right)$ for any $\mathbf{q}_{N} \in S\left(\mathbf{p}_{N}, x_{N}\right)$.

Property (i) shows that the algorithm is feasible in finite time, which is a minimal requirement for practical applicability. Next, property (ii) states that each demand $\mathbf{q}_{t}\left(\hat{x}_{t}\right)$ represents the 'highest point' on the expansion path $\mathbf{q}_{t}$ that is revealed worse than any bundle in the support set $S\left(\mathbf{p}_{N}, x_{N}\right)$.

Two further remarks are in order. First, our earlier assumptions ensure that any income level computed in Step 1 of Algorithm 1 is uniquely defined. As such, computing any set $F_{s+1}$ is straightforward. Moreover, one can show that the worst-case complexity of this algorithm is $T^{3}$, which means that the algorithm is efficiently implemented. ${ }^{7}$

Second, it is interesting to note that Algorithm 1 can also be used to extend the 'best' SARP-based test that was originally proposed by BBC (2003). ${ }^{8}$ Specifically, using information on expansion paths $\mathbf{q}_{t}(t=1, \ldots, T)$, these authors define a best possible test for SARP consistency of a particular quantity bundle $\mathbf{q}_{N}\left(x_{N}\right)$ (with $N \in\{1, \ldots$, $T\}$ ) that is conditional on some a priori defined (revealed preference) ordering of the observations. Algorithm 1 provides the basis for an alternative 'best' test: we can use the algorithm to define the set $S\left(\mathbf{p}_{N}, x_{N}\right)$, so that we can subsequently check whether $\mathbf{q}_{N}\left(x_{N}\right) \in S\left(\mathbf{p}_{N}, x_{N}\right)$ (i.e. $\mathbf{q}_{N}\left(x_{N}\right)$ is SARP consistent) or $\mathbf{q}_{N}\left(x_{N}\right) \notin S\left(\mathbf{p}_{N}, x_{N}\right)$ (i.e. $\mathbf{q}_{N}\left(x_{N}\right)$ is SARP inconsistent). It can be verified that this alternative test actually is formally identical to the one of BBC (2003), except for the important difference that it does not require a prior ordering specification - it simultaneously considers all possible (T!) orderings of the $T$ observations.

\footnotetext{
${ }^{6}$ For completeness, we add that the set $S\left(\mathbf{p}_{N}, x_{N}\right)$ is not empty, as is easily verified.

${ }^{7}$ For the sake of brevity, we do not include a formal proof of this statement here, but it is available upon request.

${ }^{8}$ BBC (2003) originally introduced a (best) test based on the Generalized Axiom of Revealed Preference (GARP) rather than SARP (which we consider here, following BBC (2008)). However, it is straightforward to adapt their ideas to obtain a best SARP-based test. See, for example, Varian (1982 and 2006) for the subtle difference between SARP and GARP.
} 


\section{Equivalent and compensating variations}

In this section, we use the results outlined above to define tightest bounds on equivalent and compensating variations. We first present formal definitions of compensating and equivalent variations. Subsequently, we show how to compute tightest bounds on these welfare measures by using our results of the previous sections.

Suppose the policy maker wants to compare two situations characterized by different price regimes: $\mathbf{p}_{O} \in \mathbb{R}_{++}^{J}$ represents original (observed; pre-reform) prices and $\mathbf{p}_{N} \in$ $\mathbb{R}_{++}^{J}$ represents new (unobserved; post-reform) prices. Income is the same in the two situations, i.e. $x_{O}=x_{N}$. Let $e(\mathbf{p}, u)$ be the expenditure function that associates minimal expenditure with prices $\mathbf{p}$ and utility $u$. By construction, rational consumer behaviour implies $e\left(\mathbf{p}_{O}, u_{O}\right)=e\left(\mathbf{p}_{N}, u_{N}\right)=x_{N}\left(=x_{O}\right)$ (where we assume that the total budget is fixed). Then, we get the following definitions.

Definition 6 (equivalent and compensating variations)

(i) Equivalent variation $E V=e\left(\mathbf{p}_{O}, u_{N}\right)-e\left(\mathbf{p}_{O}, u_{O}\right)=e\left(\mathbf{p}_{O}, u_{N}\right)-x_{N}$.

(ii) Compensating variation $C V=e\left(\mathbf{p}_{N}, u_{N}\right)-e\left(\mathbf{p}_{N}, u_{O}\right)=x_{N}-e\left(\mathbf{p}_{N}, u_{O}\right)$.

Tightest bounds for $\boldsymbol{E} \boldsymbol{V}$. To bound $E V$, we need tightest bounds on $e\left(\mathbf{p}_{O}, u_{N}\right)$. Let $e^{L}$ denote the tightest (= 'highest') lower bound and $e^{U}$ the tightest (= 'lowest') upper bound, so that $e^{L} \leq e\left(\mathbf{p}_{O}, u_{N}\right) \leq e^{U}$. The next algorithm computes $e^{L}$ and $e^{U}$. (In the algorithm, we make use of the vectors $\mathbf{P}_{j} \in \mathbb{R}^{J}$ of which all components are zero except for the $j$-th component, which equals one.)

\footnotetext{
Algorithm 2 (computing iterated bounds on $E V$ )

Input: $\left\{\mathbf{p}_{N}, x_{N}\right\}$ and $\left\{\mathbf{p}_{1}, \ldots, \mathbf{p}_{T} ; \mathbf{q}_{1}\left(x_{1}\right), \ldots, \mathbf{q}_{T}\left(x_{T}\right)\right\}$.

Output: $e^{L}$ and $e^{U}$.
}

Step 1: Use Algorithm 1 to compute the most informative incomes $\hat{x}_{1}, \ldots, \hat{x}_{T}$.

Step 2: Set $W\left(\mathbf{p}_{N}, x_{N}\right)=\varnothing$. For all $k \in\{0, \ldots, J-1\}$, consider every selection of $k$ mutually different $j_{1}, \ldots, j_{k} \in\{1, \ldots, T\}$ and $J-1-k$ mutually different $j_{k+1}, \ldots, j_{J-1} \in\{1, \ldots, J\}$. If the vectors $\mathbf{p}_{N}, \mathbf{p}_{j_{1}}, \ldots, \mathbf{p}_{j_{k}}, \mathbf{P}_{j_{k+1}}, \ldots, \mathbf{P}_{j_{J-1}}$ are linearly independent, then define the unique solution $\mathbf{q}_{N} \in \mathbb{R}^{J}$ of the system $\mathbf{p}_{N}^{\prime} \mathbf{q}_{N}=x_{N}, \mathbf{p}_{j_{1}}^{\prime} \mathbf{q}_{N}=\hat{x}_{j_{1}}, \ldots, \mathbf{p}_{j_{k}}^{\prime} \mathbf{q}_{N}=\hat{x}_{j_{k}}, \mathbf{P}_{j_{k+1}}^{\prime} \mathbf{q}_{N}=0, \ldots, \mathbf{P}_{j_{J-1}}^{\prime} \mathbf{q}_{N}=0$, and add $\mathbf{q}_{N}$ to $W\left(\mathbf{p}_{N}, x_{N}\right)$.

Step 3: Set $V\left(\mathbf{p}_{N}, x_{N}\right)=\varnothing$. Compute $V\left(\mathbf{p}_{N}, x_{N}\right)=W\left(\mathbf{p}_{N}, x_{N}\right) \cap S\left(\mathbf{p}_{N}, x_{N}\right)$.

Step 4: For every $\mathbf{q}_{N} \in V\left(\mathbf{p}_{N}, x_{N}\right)$, use Algorithm A (resp. Algorithm B) of BBC (2003) to compute $e_{\mathbf{q}_{N}}^{L}\left(\right.$ resp. $\left.e_{\mathbf{q}_{N}}^{U}\right)$.

Step 5: Set $e^{L}=\min _{\mathbf{q}_{N} \in V\left(\mathbf{p}_{N}, x_{N}\right)} e_{\mathbf{q}_{N}}^{L}$ and $e^{U}=\max _{\mathbf{q}_{N} \in V\left(\mathbf{p}_{N}, x_{N}\right)} e_{\mathbf{q}_{N}}^{U}$.

Tightest bounds for $\boldsymbol{C} \boldsymbol{V}$. To bound $C V$, we need to define bounds on $e\left(\mathbf{p}_{N}, u_{O}\right)$. Because $\mathbf{q}\left(\mathbf{p}_{O}, x_{N}\right)$ is assumed to be the observed (pre-reform) demand, we can use the bounds for the cost function $c\left(\mathbf{q}_{O}, \mathbf{p}_{N}\right)$ (that gives the minimal cost for obtaining a bundle on the same indifference curve as $\mathbf{q}_{O}$ at prices $\mathbf{p}_{N}$ - this function is equivalent to the expenditure function evaluated at the utility level $u_{O}$ generated by $\mathbf{q}_{O}$ and prices $\mathbf{p}_{N} \cdot{ }^{9}$

\footnotetext{
${ }^{9}$ The algorithms A and B of BBC (2003) can be used to obtain tightest bounds.
} 
Implementation. The algorithms are very easy-to-implement and will efficiently compute $e^{L}$ and $e^{U}$. More precisely, we can refer to our discussion on the efficiency of Algorithm 1 in the previous section, which carries over to BBC (2003)'s Algorithms A and B (which are formally similar to Algorithm 1). Next, Theorem 1 implies that the closure of $S\left(\mathbf{p}_{N}, x_{N}\right)$ is a convex set defined by linear constraints. Steps 2 and 3 of Algorithm 2 then compute the extreme points (or vertices) of this convex set. Essentially, defining each such extreme point boils down to finding the unique solution of a system with $J$ linear constraints that follow from the characterization of the convex set. That is, the budget constraint (i.e. $\mathbf{p}_{N}^{\prime} \mathbf{q}_{N}=x_{N}$ ), the constraints corresponding to a selection of $k$ observations (i.e. $\mathbf{p}_{j_{1}}^{\prime} \mathbf{q}_{N}=\hat{x}_{j_{1}}, \ldots, \mathbf{p}_{j_{k}}^{\prime} \mathbf{q}_{N}=\hat{x}_{j_{k}}$ ) and $J-1-k$ positivity constraints (i.e. $\left.\mathbf{P}_{j_{k+1}}^{\prime} \mathbf{q}_{N}=0, \ldots, \mathbf{P}_{j_{J-1}}^{\prime} \mathbf{q}_{N}=0\right) .{ }^{10}$

Some of the solutions of Step 2 do not necessarily belong to the support set $S\left(\mathbf{p}_{N}, x_{N}\right)$, which is why we need the additional Step 3 to obtain only the relevant points (i.e. the extreme points). Finally, by construction the set $V\left(\mathbf{p}_{N}, x_{N}\right)$ is finite and discrete, which implies that Step 4 of Algorithm 2 is computable in finite time.

Example 2 illustrates the different steps of Algorithm 2. The following lemma formally states that the algorithm effectively compute the tightest bounds on EV.

Lemma 2 (iterated bounds are tightest) The values $e^{L}$ and $e^{U}$ produced by Algorithm 2 define tightest bounds on $E V$.

Example 2 Figure 1 graphically illustrates the intuition behind Algorithm 2. For simplicity, we focus on a setting with only two goods and three observed price vectors (i.e. three expansion paths). The upper-left panel of the figure shows the support set $S\left(\mathbf{p}_{N}, x_{N}\right)$, which corresponds to the bold line segment. ${ }^{11}$ The set $S\left(\mathbf{p}_{N}, x_{N}\right)$ is characterized by the most informative incomes $\hat{x}_{1}, \hat{x}_{2}$ and $\hat{x}_{3}$, which are obtained through Step 1 of Algorithm 2. The corresponding set of extreme points $V\left(\mathbf{p}_{N}, x_{N}\right)=\left\{q_{N 1}, q_{N 2}\right\}$; this set is constructed in Steps 2 and 3 of Algorithm 2. The upper-right and lower-left panels of Figure 1 then show the inner and outer bounds for the indifference curves associated with, respectively, $\mathbf{q}_{N 1}$ and $\mathbf{q}_{N 2} \cdot{ }^{12}$ In turn, this defines the lower bounds $e_{\mathbf{q}_{N 1}^{L}}^{L}$ and $e_{\mathbf{q}_{N 2}}^{L}$ and the upper bounds $e_{\mathbf{q}_{N 1}}^{U}$ and $e_{\mathbf{q}_{N 2}}^{U}$, which are generated in Step 4 of Algorithm 2. Finally, the lower-right panel of Figure 1 shows the resulting values of $e^{L}$ and $e^{U}$, which are obtained in Step 5 of Algorithm 2. Here, we have $e^{L}=\min \left\{e_{\mathbf{q}_{N 1}}^{L}, e_{\mathbf{q}_{N 2}}^{L}\right\}=e_{\mathbf{q}_{N 1}}^{L}=$ $e_{\mathbf{q}_{N 2}}^{L}$ and $e^{U}=\max \left\{e_{\mathbf{q}_{N 1}}^{U}, e_{\mathbf{q}_{N 2}}^{U}\right\}=e_{\mathbf{q}_{N 1}}^{U}$.

\section{An illustration}

To illustrate the application of these methods we performed the following simple exercise. We took the data on prices, mean budget shares and expenditures from Table A.1 in the Appendix in BBC (2008); see our Appendix B for more details. These data relate to their analysis of three broad consumption groups: food, other nondurables, and

\footnotetext{
${ }^{10}$ Although Step 2 is directly implementable, we also note that it should not be the most efficient way to compute the extreme points of our convex set. Indeed, given that this set is characterized by linear constraints, computing these extreme points is equivalent to finding all basic feasible solutions of a system of linear equations. Alternative algorithms for computing these basic feasible solutions are available in the Operations Research literature.

${ }^{11}$ Since there are only two goods in this example, the support set $S\left(\mathbf{p}_{N}, x_{N}\right)$ actually coincides with BBC's support set $S^{B B C}\left(\mathbf{p}_{N}, x_{N}\right)$, which are characterized by intersection demands (see Proposition 1). (Correspondingly, the most informative incomes $\hat{x}_{1}, \hat{x}_{2}$ and $\hat{x}_{3}$ equal the intersection incomes $\widetilde{x}_{1}$, $\widetilde{x}_{2}$ and $\left.\widetilde{x}_{3}.\right)$ As explained above, the sets $S\left(\mathbf{p}_{N}, x_{N}\right)$ and $S^{B B C}\left(\mathbf{p}_{N}, x_{N}\right)$ need not coincide in case there are more than two goods. We choose to focus on a two-goods setting here as this allows us to better illustrate the mechanics of Algorithm 2.

12 These bounds for indifference curves are (implicitly) constructed in Algorithms A and B of BBC (2003). We refer to these authors for a detailed discussion on the construction method. See in particular their Figure 7.
} 

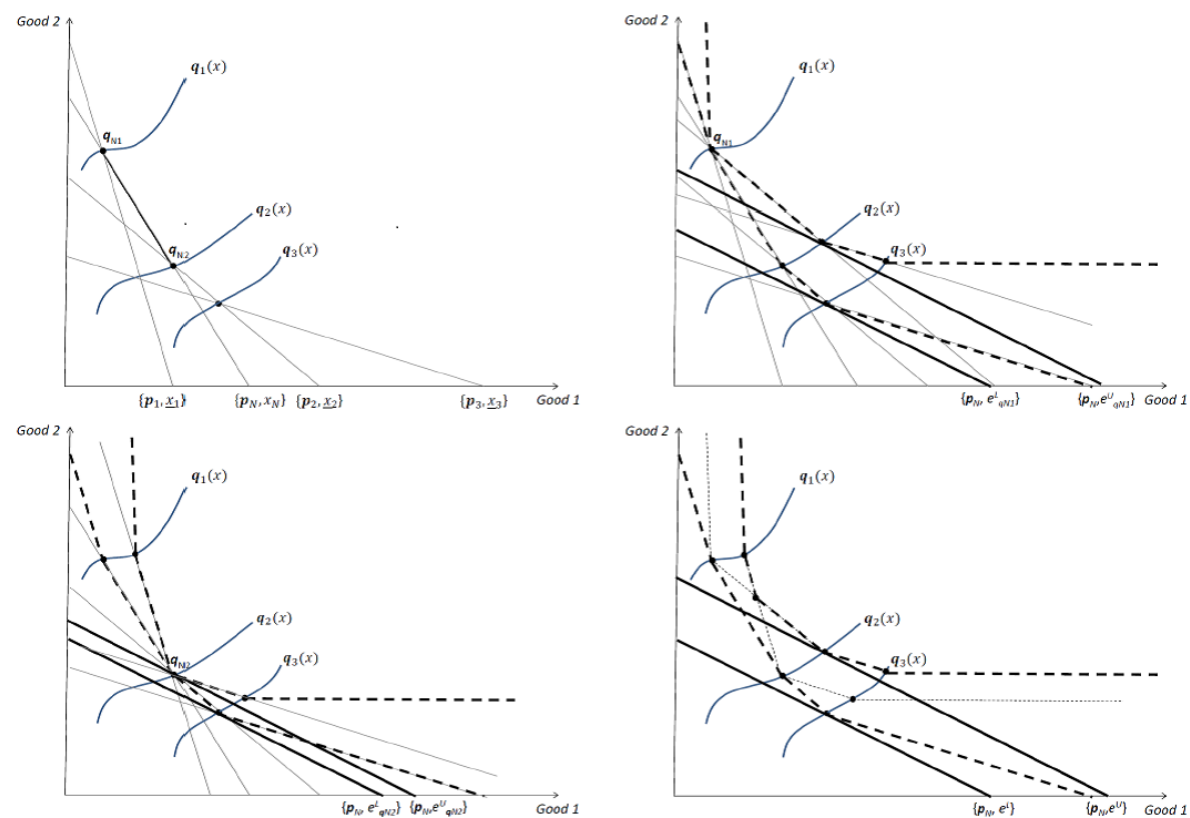

Figure 1: Illustration of Algorithm 1

services. ${ }^{13}$ Rather than re-estimate their semi-parametric Engel curves in full we relied, purely for simplification, on the heuristic assumption that for small changes in income budget shares are approximately flat. To validate this, we took the data from Table B.1 and carried out a test of the Homothetic Axiom of Revealed Preference (HARP, see Varian, 1983, Theorem 2). This was satisfied. We emphasise that local homotheticity is just an assumption-of-convenience, made for the sake of simplifying the illustration of the methods described here. Whilst the data at mean budget shares and expenditures does indeed survive a test of HARP, we do not wish to lean on this assumption too much and therefore, in order to keep the income variation required as small as possible, we selected a contiguous subset of periods from the data roughly in the middle of the observed scatterplot of relative prices, which related to the years 1985 to $1988 .{ }^{14}$ This ensured that it is not necessary to move too far along any of the Engel curves whilst applying either Algorithm 1 or 2. Whilst dropping observations might widen the bounds on predicted demand relative to what might be possible if we kept all of the data, as we shall see below these other observations turn out to be rarely informative and consequently the bounds on predicted demands are very tight even without them.

We then considered a new budget $\left\{\mathbf{p}_{0}, x_{0}\right\}$ where the price vector and the new total expenditure were set equal to the mean of the data for our sub-period of interest, and calculated both the i-bounds and the e-bounds on the predicted budget shares for the three goods. The results are illustrated in Figure 2.

Figure 2 shows the budget unit simplex for the predicted budget shares (the three corners refer to bundles in which the consumer exhausts her entire budget on a single good). A little below the centre is a small area which illustrates the i-bounds and the e-bounds on the predicted budget shares. The first thing to notice is that this set is

\footnotetext{
${ }^{13}$ They used 25 years of British Family Expenditure Surveys from 1975 to 1999. The Family Expenditure Survey (FES) is a repeated cross-section survey consisting of around 7,000 households in each year. From these data they drew the sub-sample of couples with children who own a car. This gives us between 1,421 and 1,906 observations per year and 40,731 observations over the entire period. They use total spending on non-durables to define the total expenditure variable. Table A1 in the Data Appendix of BBC (2008) provides descriptive statistics for these data.

${ }^{14}$ See Figure 3 in $\mathrm{BBC}(2008)$ for more details.
} 


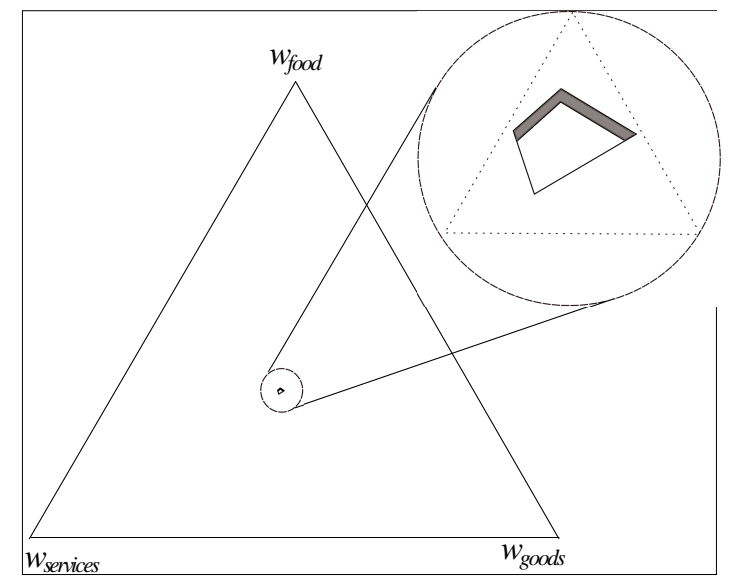

Figure 2: i-bounds and e-bounds on predicted budget shares

very small - we have provided a magnified view of it so that its shape can be seen more clearly. The bounds on the budget shares for each commodity group are of the order of 1 to 2 percentage points across. This precisely mirrors the result in BBC (2008) - see Figures 4 to 6 - which showed that when the new budget lies within the convex hull of the observed budgets, it is possible to recover highly informative bounds on predicted behaviour using just RP restrictions.

The second point to notice can be seen from the magnified view of the i-bound and e-bound sets. The e-bounds are the entire trapezoidal area (both the white and shaded areas). The i-bounds, which exploit transitivity, are shown by the white portion of this area only. Points in the shaded area satisfy WARP in combination with the rest of the data. Points in the white area additionally satisfy SARP. Note that using SARP improves the bounds in all three dimensions. Comparing the white area with the shaded area, shows that the iterative procedure brings an improvement of approximately $11 \%$.

Finally, we also calculated bounds on the welfare effects of the change in prices from their observed level in 1986 to the new price vector (we chose 1986 as the base as prices in this period were the furthest, by Euclidean distance, from the new prices). Overall the change in the budget was such that both average prices and the budget rose but a little under one percent so, whilst prices rose, the consumer's nominal income rose too. We found:

$$
\begin{aligned}
& E V=e\left(\mathbf{p}_{86}, u_{N}\right)-e\left(\mathbf{p}_{86}, u_{86}\right) \in[0.058163,0.061531] \\
& C V=e\left(\mathbf{p}_{N}, u_{N}\right)-e\left(\mathbf{p}_{N}, u_{86}\right) \in[0.058647,0.06035] .
\end{aligned}
$$

For comparison we note that $x_{86}=38.41$ and $x_{N}=38.789$. That the welfare effects are small in magnitude should therefore not be very surprising given that one would expect a one percent change in prices to be roughly compensated by a one percent change in income. We are however mainly concerned with the tightness of the bounds. As expected the bounds on the $E V$ are somewhat wider than those for the $C V$ because it is necessary to bound the indifference curve through the predicted demand rather than the observed (base) demand. However, the tightness of the $E V$ bounds is gratifying and reflects the tightness of the bounds we are able to place on the uncompensated demand response. 


\section{Conclusion}

In this paper we have refined and extended the results of Blundell, Browning and Crawford (2003, 2008). We defined tightest "iterated" (nonparametric) bounds on counterfactual Marshallian demands that apply to any number of goods. These bounds are sharp under the Strong Axiom of Revealed Preference, SARP. We were thus able to show they provide sharp bounds for counterfactual welfare measures.

We have established a complete toolkit for a powerful nonparametric welfare analysis based on Hicksian compensating and equivalent variations. We further showed that our iterated bounds method involves computational algorithms that are easily implemented. Finally, we presented an empirical illustration of the practical usefulness of our results.

\section{Appendix A. Proofs}

\section{Proof of Proposition 1}

By construction we have $\mathbf{q}_{N} R^{0} \mathbf{q}_{t}\left(\tilde{x}_{t}\right)$ for any $\mathbf{q}_{N} \in B\left(\mathbf{p}_{N}, x_{N}\right)$. If $\mathbf{q}_{N} \in S^{B B C}\left(\mathbf{p}_{N}, x_{N}\right)$ and $\mathbf{q}_{N} \neq \mathbf{q}_{t}\left(\tilde{x}_{t}\right)$ for all $t$, then SARP consistency for $\mathbf{q}_{N} R^{0} \mathbf{q}_{t}\left(\tilde{x}_{t}\right)$ implies that $\mathbf{p}_{t}^{\prime} \mathbf{q}_{t}\left(\tilde{x}_{t}\right)<$ $\mathbf{p}_{t}^{\prime} \mathbf{q}_{N}$ (i.e. not $\left.\mathbf{q}_{t}\left(\tilde{x}_{t}\right) R^{0} \mathbf{q}_{N}\right)$. Next, if $\mathbf{q}_{N}=\mathbf{q}_{s}\left(\tilde{x}_{s}\right) \in S^{B B C}\left(\mathbf{p}_{N}, x_{N}\right)$ for some $s$, then SARP consistency requires the same for all observations $t \neq s$.

Conversely, take any $\mathbf{q}_{N} \in B\left(\mathbf{p}_{N}, x_{N}\right)$, then $\mathbf{p}_{t}^{\prime} \mathbf{q}_{t}\left(\tilde{x}_{t}\right)<\mathbf{p}_{t}^{\prime} \mathbf{q}_{N}$ for all $t$ (i.e. condition (i) holds) excludes $\mathbf{q}_{t}\left(\tilde{x}_{t}\right) R \mathbf{q}_{N}$. So a rejection of SARP requires $\mathbf{q}_{N} R \mathbf{q}_{t}\left(\tilde{x}_{t}\right)$ and $\mathbf{p}_{t}^{\prime} \mathbf{q}_{t}\left(\tilde{x}_{t}\right) \geq \mathbf{p}_{t}^{\prime} \mathbf{q}_{N}$ (i.e. $\left.\mathbf{q}_{t}\left(\tilde{x}_{t}\right) R^{0} \mathbf{q}_{N}\right)$. But this last inequality is excluded by assumption, and thus $\mathbf{q}_{N} \in S^{B B C}\left(\mathbf{p}_{N}, x_{N}\right)$. A similar reasoning holds for $\mathbf{q}_{N}=\mathbf{q}_{s}\left(\tilde{x}_{s}\right)$ (i.e. if condition (ii) holds).

\section{Proof of Theorem 1}

Suppose $\mathbf{q}_{N} \in S\left(\mathbf{p}_{N}, x_{N}\right)$ and $\mathbf{q}_{N} \neq \mathbf{q}_{t}\left(\widehat{x}_{t}\right)$ for all $t$. By construction we have that $\mathbf{q}_{N} R \mathbf{q}_{t}\left(\widehat{x}_{t}\right)$ for any $\mathbf{q}_{N} \in B\left(\mathbf{p}_{N}, x_{N}\right)$. So SARP consistency requires that $\mathbf{p}_{t}^{\prime} \mathbf{q}_{t}\left(\widehat{x}_{t}\right)<$ $\mathbf{p}_{t}^{\prime} \mathbf{q}_{N}$. Next, assume that $\mathbf{q}_{N}=\mathbf{q}_{s}\left(\widehat{x}_{s}\right) \in S\left(\mathbf{p}_{N}, x_{N}\right)$ for some $s$. Then, SARP consistency requires the same for all $t \neq s$.

Conversely, take any $\mathbf{q}_{N} \in B\left(\mathbf{p}_{N}, x_{N}\right)$ and suppose that $\mathbf{p}_{t}^{\prime} \mathbf{q}_{t}\left(\widehat{x}_{t}\right)<\mathbf{p}_{t}^{\prime} \mathbf{q}_{N}$ for all $t$ (i.e. condition (i) holds). Then, normality implies for all $x_{t} \leq \widehat{x}_{t}$ that $\mathbf{p}_{t}^{\prime} \mathbf{q}_{t}\left(x_{t}\right) \leq \mathbf{p}_{t}^{\prime} \mathbf{q}_{t}\left(\widehat{x}_{t}\right)<$ $\mathbf{p}_{t}^{\prime} \mathbf{q}_{N}$. Therefore, by Definition 5 and the above, we cannot have $\mathbf{q}_{t}\left(x_{t}\right) R \overline{\mathbf{q}}_{N}$. As such, there can be a rejection of SARP only if, for some income $x_{t}$, we have $\mathbf{q}_{N} R \mathbf{q}_{t}\left(x_{t}\right)$ and $\mathbf{p}_{t}^{\prime} \mathbf{q}_{t}\left(x_{t}\right) \geq \mathbf{p}_{t}^{\prime} \mathbf{q}_{N}$. Suppose, then, that we do have such a rejection, i.e. there exists an income $x_{t}$ for which $\mathbf{q}_{N} R \mathbf{q}_{t}\left(x_{t}\right)$ and $\mathbf{p}_{t}^{\prime} \mathbf{q}_{t}\left(x_{t}\right) \geq \mathbf{p}_{t}^{\prime} \mathbf{q}_{N}$. Since $\mathbf{p}_{t}^{\prime} \mathbf{q}_{t}\left(\widehat{x}_{t}\right)<\mathbf{p}_{t}^{\prime} \mathbf{q}_{N}$, normality implies that $x_{t}>\widehat{x}_{t}$. This gives us the wanted contradiction, since Definition 5 and the above then exclude $\mathbf{q}_{N} R \mathbf{q}_{t}\left(x_{t}\right)$.

A similar reasoning holds for $\mathbf{q}_{N}=\mathbf{q}_{s}\left(\widehat{x}_{s}\right)$ (i.e. condition (ii) holds), which finishes the proof.

\section{Proof of Lemma 1}

Algorithm 1 is formally similar to Algorithm B of BBC (2003). In particular, Step 2 of these authors' Algorithm B considers problems of the type $\max _{x_{t} \in F_{s}}\left(\mathbf{q}_{k}(x): x_{t}=\mathbf{p}_{t}^{\prime} \mathbf{q}_{k}(x)\right)$, while Step 2 of our Algorithm 1 uses $\max _{x_{t} \in F_{s}}\left(x: x_{t}=\mathbf{p}_{t}^{\prime} \mathbf{q}_{k}(x)\right)$. Because of weak normality (Assumption 2), we have that

$$
\max _{x_{t} \in F_{s}}\left(x: x_{t}=\mathbf{p}_{t}^{\prime} \mathbf{q}_{k}(x)\right)=\arg \max _{x}\left(\max _{x_{t} \in F_{s}}\left(\mathbf{q}_{k}(x): x_{t}=\mathbf{p}_{t}^{\prime} \mathbf{q}_{k}(x)\right)\right) .
$$

Given this, we can straightforwardly adapt the proof of BBC (2003)'s Propositions 3 to obtain the result in Lemma 1. 


\section{Proof of Lemma 2}

To bound EV and thus $e\left(\mathbf{p}_{O}, u_{N}\right)$, we need to find, for any $\mathbf{q}_{N} \in S\left(\mathbf{p}_{N}, x_{N}\right)$, the nonparametrically constructed 'revealed-preferred' set $R P\left(\mathbf{q}_{N}\right)$, which contains all bundles to which $\mathbf{q}_{N}$ is preferred to, and the 'not-revealed-worse' set $N R W\left(\mathbf{q}_{N}\right)$, which contains all bundles that are not revealed worse to $\mathbf{q}_{N}$. (See Varian (1982) for an extensive discussion of the sets $R P\left(\mathbf{q}_{N}\right)$ and $N R W\left(\mathbf{q}_{N}\right)$.)

Given our results in Section 2, we can define tightest bounds on EV by computing $e_{\mathbf{q}_{N}}^{L}\left(\operatorname{resp} . e_{\mathbf{q}_{N}}^{U}\right)$ for any $\mathbf{q}_{N} \in S\left(\mathbf{p}_{N}, x_{N}\right)$. Now, Proposition 1 implies that the closure of $S\left(\mathbf{p}_{N}, x_{N}\right)$ is a convex set and, as discussed in the main text, $V\left(\mathbf{p}_{N}, x_{N}\right)$ contains all the extreme points of this convex set. As such, we get that any $\mathbf{q}_{N} \in S\left(\mathbf{p}_{N}, x_{N}\right)$ can be written as a convex combination of elements of $V\left(\mathbf{p}_{N}, x_{N}\right)$, i.e. $\mathbf{q}_{N}=\sum_{k} \lambda_{k} \mathbf{q}_{k}$ (with $\lambda_{k}>0$ and $\left.\sum_{k} \lambda_{k}=1\right)$ for $\mathbf{q}_{k} \in V\left(\mathbf{p}_{N}, x_{N}\right)$.

Given this, and using convexity of preferences (represented by the sets $R P\left(\mathbf{q}_{N}\right)$ and $\left.N R W\left(\mathbf{q}_{N}\right)\right)$, we get $R P\left(\mathbf{q}_{N}\right) \subseteq R P\left(\mathbf{q}_{k}\right)$ for at least one $\mathbf{q}_{k} \in V\left(\mathbf{p}_{N}, x_{N}\right)$ and also that $N R W\left(\mathbf{q}_{N}\right) \subseteq N R W\left(\mathbf{q}_{k}\right)$ for at least one, possibly different, $\mathbf{q}_{k} \in V\left(\mathbf{p}_{N}, x_{N}\right)$. As such, in order to nonparametrically identify the lower bound $e^{L}$ (respectively, upper bound $e^{U}$ ), we need to take the minimum (respectively, maximum) of the lower (respectively, upper) bounds over all the elements of $V\left(\mathbf{p}_{N}, x_{N}\right)$, i.e. $e^{L}=\min _{\mathbf{q}_{N} \in V\left(\mathbf{p}_{N}, x_{N}\right)} e_{\mathbf{q}_{N}}^{L}$ and $e^{U}=\max _{\mathbf{q}_{N} \in V\left(\mathbf{p}_{N}, x_{N}\right)} e_{\mathbf{q}_{\mathbf{N}}}^{U}$. 


\section{Appendix B. Descriptive statistics}

Table B1. Descriptive Statistics

\begin{tabular}{cccccccc}
\hline \hline \multicolumn{6}{c}{ Budget Shares } & Total Exp. & \multicolumn{2}{c}{ Prices } & Obs. \\
& $\mathrm{F}$ & ND & $\mathrm{S}$ & & $\mathrm{F}$ & $\mathrm{S}$ & \\
\hline 1975 & 0.3587 & 0.3166 & 0.3247 & 33.7838 & 1.0000 & 1.0000 & 1873 \\
1976 & 0.3577 & 0.3076 & 0.3347 & 32.5127 & 1.0881 & 1.0687 & 1642 \\
1977 & 0.3564 & 0.3124 & 0.3312 & 32.3477 & 1.1574 & 1.0447 & 1770 \\
1978 & 0.3556 & 0.3136 & 0.3308 & 32.5452 & 1.1067 & 1.0398 & 1681 \\
1979 & 0.3458 & 0.3196 & 0.3346 & 36.4990 & 1.1457 & 1.0414 & 1689 \\
1980 & 0.3384 & 0.3208 & 0.3408 & 36.6857 & 1.1145 & 1.1061 & 1781 \\
1981 & 0.3363 & 0.3061 & 0.3576 & 35.7316 & 1.1056 & 1.1836 & 1906 \\
1982 & 0.3218 & 0.3101 & 0.3681 & 35.8705 & 1.1262 & 1.2199 & 1876 \\
1983 & 0.3214 & 0.3129 & 0.3657 & 35.6571 & 1.0775 & 1.2429 & 1743 \\
1984 & 0.3162 & 0.3151 & 0.3688 & 37.5016 & 1.1081 & 1.2492 & 1671 \\
1985 & 0.3081 & 0.3207 & 0.3712 & 37.8100 & 1.0759 & 1.2242 & 1622 \\
1986 & 0.3088 & 0.3221 & 0.3692 & 38.4100 & 1.0556 & 1.2239 & 1587 \\
1987 & 0.3043 & 0.3228 & 0.3730 & 39.0197 & 1.0819 & 1.2372 & 1632 \\
1988 & 0.3042 & 0.3278 & 0.3680 & 41.5325 & 1.0807 & 1.2512 & 1648 \\
1989 & 0.3054 & 0.3222 & 0.3724 & 41.5346 & 1.0786 & 1.2713 & 1652 \\
1990 & 0.3017 & 0.3129 & 0.3854 & 44.2983 & 1.1084 & 1.3150 & 1538 \\
1991 & 0.2972 & 0.3103 & 0.3925 & 42.6966 & 1.0839 & 1.3207 & 1510 \\
1992 & 0.2882 & 0.3121 & 0.3997 & 41.5212 & 1.0616 & 1.3445 & 1578 \\
1993 & 0.2866 & 0.3077 & 0.4057 & 41.3798 & 1.0332 & 1.3533 & 1511 \\
1994 & 0.2825 & 0.3029 & 0.4146 & 40.9660 & 1.0305 & 1.3748 & 1489 \\
1995 & 0.2912 & 0.2912 & 0.4176 & 39.6002 & 1.0439 & 1.3645 & 1502 \\
1996 & 0.2889 & 0.2999 & 0.4112 & 41.8850 & 1.0671 & 1.3491 & 1476 \\
1997 & 0.2741 & 0.3041 & 0.4218 & 45.2517 & 1.0655 & 1.4071 & 1421 \\
1998 & 0.2788 & 0.2981 & 0.4230 & 44.0626 & 1.0551 & 1.4102 & 1432 \\
1999 & 0.2722 & 0.3032 & 0.4245 & 47.1033 & 1.0918 & 1.4367 & 1501 \\
\hline \hline Notes: F=Food, ND=Non-durables,S=Services & & & & \\
& & & & & & &
\end{tabular}




\section{References}

[1] Afriat, S. (1967). "The construction of utility functions from expenditure data", International Economic Review, 8, 67-77.

[2] Banks, J., R. Blundell and A. Lewbel (1997), "Quadratic Engel curves and consumer demand", Review of Economics and Statistics, 79, 527-539.

[3] Blundell, R. (2005), "How revealing is revealed preference?", Journal of the European Economic Association, 3, 211-235.

[4] Blundell, R., M. Browning and I. Crawford (2003), "Nonparametric Engel curves and revealed preference", Econometrica, 71, 205-240.

[5] Blundell, R., M. Browning and I. Crawford (2007), "Improving revealed preference bounds on demand responses", International Economic Review, 48n 1227-1244.

[6] Blundell, R., M. Browning and I. Crawford (2008), "Best nonparametric bounds on demand responses", Econometrica, 76, 1227-1262.

[7] Blundell, R., D. Kristensen and R. Matzkin (2013), "Bounding quantile demand functions using revealed preference inequalities", Journal of Econometrics, forthcoming.

[8] Diewert, E. (1973), "Afriat and revealed preference theory", Review of Economic Studies, 40, 419-425.

[9] Hicks, J. (1939), Value and Capital. An Inquiry into some Fundamental Principles of Economic Theory, Oxford University Press, Oxford.

[10] Houthakker, H. (1950), "Revealed preference and the utility function", Economica, $17,159-174$.

[11] Kihlstrom, R., A. Mas-Colell and H. Sonnenschein (1976), "The demand theory of the weak axiom of revealed preference", Econometrica, 44, 971-978.

[12] Rose, H. (1958), "Consistency of preference: the two-commodity case", Review of Economic Studies, 25, 124-125.

[13] Samuelson, P. (1938). "A note on the pure theory of consumer's behaviour", Economica, vol. 5(17), pp. 61-71.

[14] Samuelson, P. (1948). "Consumption theory in terms of revealed preference", Economica, vol. 15(60), pp. 243-253.

[15] Samuelson, P. (1974), "Complementarity: an essay on the 40th anniversary of the Hicks-Allen revolution in demand theory", Journal of Economic Literature, 12, 1255-1289. 
[16] Varian, H. (1982). "The nonparametric approach to demand analysis", Econometrica, vol. 50(4), pp. 945-972.

[17] Varian, H. (2006), "Revealed preference", in M. Szenberg, L.Ramrattan and A. Gottesman (eds.), Samuelsonian Economics and the 21st Century, Oxford University Press. 